\title{
Developing Models for Managing Drones in the Transportation System in Smart Cities
}

\author{
Nguyen Dinh Dung" (Ph.D. student, Department of Aeronautics, Naval Architecture and Railway Vehicles, \\ Budapest University of Technology and Economics, Hungary)
}

\begin{abstract}
Unmanned aerial vehicles (UAVs), especially drones, have advantages of having applications in different areas, including agriculture, transportation, such as land use surveys and traffic surveillance, and weather research. Many network protocols are architected for the communication between multiple drones. The present study proposes drone-following models for managing drones in the transportation management system in smart cities. These models are based on the initial idea that drones flight towards a leading drone in the traffic flow. Such models are described by the relative distance and velocity functions. Two types of drone-following models are presented in the study. The first model is a safe distance model (SD model), in which a safe distance between a drone and its ahead is maintained. By applying the stochastic diffusion process, an improved model, called Markov model, is deduced. These drone-following models are simulated in a 2D environment using numerical simulation techniques. With the simulation results, it could be noted that: i) there is no accident and no unrealistic deceleration; ii) the velocity of the followed drone is changed according to the speed of the drone ahead; iii) the followed drones keep a safe distance to drone ahead even the velocities are changed; iv) the performance of the Markov model is better than that of the SD model.
\end{abstract}

Keywords - Air transportation; Mathematical model; Path planning; Safety management; Vehicle routing.

\section{INTRODUCTION}

Drones are regularly recognised as unmanned aerial vehicles (UAVs), which are exponentially growing over the past two decades. Many research papers have been reported in the field of UAVs and their applications.

Drones are gaining more popularity in the commercial sector, and have found applications in many areas. For example, drones are used to detect areas of weeds and support for herbicides and fertilizers sprayed out over a poor yield [1]. In this way, farmers not only save a lot of money but also prevent touching the chemicals. Besides, a drone is developed, which allows operators to monitor pollution, the weather and climate, especially hazardous areas like volcanoes [2]. Moreover, film companies use drones to capture aerial shots that one may see in the latest films and TV programs [3].

Another application of drones strives to satisfy the transportation manager's requirement for collecting trajectory data from main roads, in which drones have been used as a "bird's eye view" [4]. This application is considered a novel and cost-effective solution for traffic management. Thiels et al. proposed a package delivery system based on UAVs to transport medical products [5]. This system is expected to be an effective solution for transportation. However, the authors did not show the saved cost of this program compared with a conventional approach, although it seemed to be significantly lower.

At present, drones have been developed for their applications in smart cities, which can be reviewed in academic papers [6][10]. With the need for delivering services more rapidly and more efficiently to residents, drones are the best resolution because they have an enormous quantity of potential to help cities better serve the residents [6]. Drones are not only being used to improve city life but also are expected to increase significantly. However, this potential trend would bring both advantages and new challenges for theoretical and practical aspects.

Nowadays, drones have become an essential factor in connected smart cities [7]. Several aspects of drones regarding privacy and safety, as well as cybersecurity, were presented.

$\mathrm{Wu}$ et al. mentioned the concept of a swarm of UAVs that were used for urban sensing around the city [8]. However, this approach may raise new challenges of using and managing drones in the traffic flow, such as collision avoidance and navigation. Several studied reports relating to these challenges can be found in [9], [10].

Besides, the delivery services must be quicker and more efficient to meet the demands of smart citizens. These demands can be satisfied by applying drones for delivering packages [11]. In this way, the customers may receive the purchased item within minutes, and the delivery cost is less than that provided by the traditional logistic networks.

It is expected that commercial drones will increase in the future due to their increase in recent years. For example, drones were introduced as drone-enabled services in 2015, in which the revenue was a more significant opportunity and would grow to $\$ 8.7$ billion annually by 2025 [12]. The Federal Aviation Administration (FAA) also predicted that 30000 drones could be flying in U.S. skies in the near future [13].

However, the larger the number of operating drones, the more accidents in the sky. It not only endangers civil aviation regarding security and infrastructure but also decreases traffic safety. Therefore, it is necessary to manage drones in traffic flows. The present study proposes a novel approach for the management of drones called the drone-following models. Such models are based on the initial idea that drones flight towards a

\footnotetext{
* Corresponding author.

E-mail: ddnguyen@vrht.bme.hu
} 
leading drone in the traffic flow. A relative distance and velocity functions are used to describe such models. As the best of our knowledge, this is the first study that presents a new approach for managing a group of drones in smart cities.

The goal of the study is to present a method for managing drones in the air transport system, which is based on the dronefollowing models. The approach uses the follow-the-leader theory that is applied to dense traffic flow.

Having discussed applications of drones in the transportation system, Section II provides an overview of the related studies. The formation of drone-following models is described in Section III. Section IV presents the numerical simulation results and discussion. The paper ends with a conclusion and recommendation for future studies.

\section{RELATED WORK}

In this section, several scientific reports regarding the management of drones in smart cities are discussed. The concept of a drone is provided in Section I. However, its operation must comply with the regulations of the International Civil Aviation Organization (ICAO) [14].

According to the FAA, the area for operating drones is classes of activities or specific operations that are usually prohibited for commercial drone applications. However, the airspace and equipment approval can be achieved depending on who you are and how you want to fly. This procedure is more straightforward and more flexible for operating commercial drones. Besides, a "phased-in approach" has been carried out for the integration of drones with a national airspace system. Moreover, the drones can be used for specific applications in restriction areas, for example, atmospheric research.

All drones must be managed and controlled as aircraft according to the FAA regulations [11], which are categorised as follows: 1) Model Aircraft operators; 2) Those holding 333 Exemptions; 3) Public Operators; 4) Public operators can choose to operate under Part 107.

In Europe, while the Joint Aviation Authorities (JAS) is responsible for operations and licensing, the European Aviation Safety Agency (EASA) is responsible for regulating airworthiness and maintenance issues [15].

In the aviation industry, especially the air traffic control, the increasing number of drones poses new challenges, which endanger the flights operated in the airspaces. Therefore, a unique operating aspect has to be created to protect the regular flights regarding safety and implementation. Sandor proposed the UAV traffic management systems (UTM) that supported the accomplishment of the flight for managing the total air traffic efficiently [16]. Such a system can be used to keep the separation between the UAVs and the conventional aircraft as well as the order in the traffic flow in the very low-level airspace segments. This system is operated independently of the air traffic management systems (ATM) due to the data coming to this system.

Based on the scientific results obtained from the creation of an extensive road network model, the authors in [17] proposed a new air traffic model, which was a powerful new tool in the field of air traffic network modelling and had all the specialties.
Such a system is considered as a large stochastic dynamic system that can be used to describe the processes of land traffic. As the traffic network is very complex and characterized by different rules, geometric data, seasonality, the authors developed a new model for the air traffic system. The development of a new model became possible, which led to non-linear systems in the field of mathematics. The result from the control point of view was shown by applying the method of Lyapunov function in a domain bounded by an arbitrary closed curve. The authors used static route parameters to achieve minimised delays in the field of air traffic control. The model used in the research was a macroscopic model, and it was based on a linear time-invariant homogeneous differential equation system. The optimisation objective of this model was the minimisation of total delay; for example, the landing of the aircraft should take place in the shortest time utilising the appropriate choice of control parameters. Thus, the solution of significant network problems and the application of new control options were obtained. For instance, it would sometimes occur that two airplanes flying close to each other along the same route would want to travel at the same altitude. In this case, the air traffic controller who wants to keep the required separation offers level change primarily. If it is done, there is no need for speed restriction. If the other flight levels or altitudes are occupied, then the crew may be asked to maintain a certain speed.

A case of studies regarding the legal use of drones is presented in [18], which has its perspective in the Slovak Republic. Generally, the increase in the use of drones will generate a new competitive environment for operating companies as well as cooperative enterprises. However, the recent legislation regarding drone applications does not satisfy this challenge due to the flexible legal use of drones, such as monitoring of employees and delivering of documents.

Another scientific report provided a summary of drone applications for managing transportation [19]. The authors focused on the theory and practice of UAS in transportation and traffic engineering. This survey indicated that the use of drones in transport must guarantee safety and energy efficiency.

Based on the literature review above, it can be noted that the management of drones is a significant issue not only in the transportation system but also in the air traffic system in smart cities.

To address this issue in the transportation system, the present paper proposes the methodology, called drone-following models, to manage drones in smart cities.

\section{FORMULATION OF DRONE-FOLLOWING MODELS}

In this section, we propose drone-following models. This approach is based on the theory that has been developed as a mathematical description of traffic flow. The states of drones are determined from the traffic flow data by identifying the coefficients in the equation system of motion. According to the theory, two drone-following models are proposed. The first model is used to keep safe velocity according to the related position of drones, while the second one describes the situation that a safe distance between two drones is maintained due to 
relative velocities. These models also represent the one-by-one following process of drones in the traffic flow, which can be considered as one kind of microscopic model in the transportation system. In addition, the drone-following models are expected to play essential roles in the development of intelligent transport systems and collision avoidance.

A structure of the drone-following model is described in Fig. 1. The state of the followed drone is determined by its characteristics and velocity, relative distance to the drone ahead, controller parameters, as well as weather conditions. Therefore, the followed drone's velocity and position are mostly calculated from the traffic situation related to the velocity and position of the drone ahead.

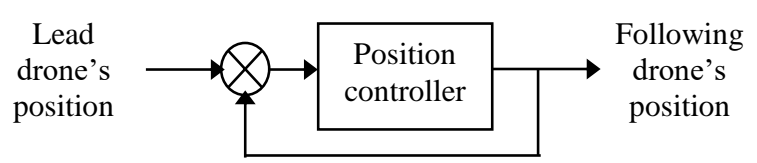

Fig. 1. A structure of the drone-following model.

The drone-following models are proposed for the following purposes:

i) producing the realistic speed profiles;

ii) ability for generating the real traffic streams;

iii) stable realisation of the traffic situations;

iv) simulation of traffic situations implemented by different combination of drones and controller parameters;

v) ability to apply them in traffic control systems.

\section{A. The Safe Distance Model}

The first drone-following model is based on the principle that keeps a safe distance according to relative velocity (SD model). Such a model describes situations that the drone's velocity depends on the traffic situation, namely on the distance to the drone ahead and its velocity. This approach has led to the linear models assuming that the acceleration of the followed drone is controlled by its controller to keep zero relative velocity to the drone ahead.

$$
m_{n} \ddot{X}_{n}(t+T)=\lambda\left[\dot{X}_{n-1}(t)-\dot{X}_{n}(t)\right],
$$

where $m_{n}$ - the mass of $n$-th drone;

$X_{n}(t+T)$ - the acceleration of $n$-th drone after a reaction;

$X_{n-1}(t)-X_{n}(t)$ - relative velocity of $(n-1)$-th to the $n$-th drone in time $t$;

$T$ - delay time of a controller;

$\Lambda$ - a weight coefficient related to the controllers.

Then, the SD model is given as follows:

$$
\ddot{X}_{n}(t+T)=\lambda \frac{\left[\dot{X}_{n}(t)\right]^{p}}{\left[X_{n-1}(t)-X_{n}(t)\right]^{q}}\left[\dot{X}_{n-1}(t)-\dot{X}_{n}(t)\right],
$$

where $p, q$-parameters related to velocity and distance of the drone ahead;

$X_{n-1}(t)-X_{n}(t)$ - relative distance between the $(n-1)$-th drone and the $n$-th drone.
It can be noted that (2) describes a steady state that satisfies the small velocity disturbances. Besides, the relative distance between drones contributes to (2) in matching experimental data. However, the spacing term is essential in mathematical models based on an optimal control approach. This approach is to formulate an algorithm as a model of the following system, in which a safe distance between drones will be satisfied. It is expected that the method is more efficient when the drones fly in a row in the traffic flow.

If the model is used for a simulation environment, the historical data, such as acceleration, velocity, and position of drones, will be stored for successive recalculations. Besides, it can be assumed that when the leading drone is held suddenly, the followed drone can maintain safety because of its controlled velocity.

The SD model is deduced by setting limits on the performance of controller and drone and using these limits to calculate a safe distance related to the leading drone. Such a model has the following characteristics:

i) the speed of the followed drone will accelerate if the drone ahead accelerates;

ii) the velocity of the followed drone will decrease if the drone ahead decelerates.

This model will be evaluated in a numerical environment with a small-time increment. The state of the drone ahead can be changed at each time increment. For example, if its velocity accelerates at a given rate at time $t$, then it accelerates at another rate at the next time $t+\Delta t$. The velocity and position of the followed drone are then updated after each time increment.

\section{B. The Markov Model}

The SD model is used and improved for different traffic situations in a numerical simulation environment. However, this model has two disadvantages:

i) the coefficients applied in the models intensely depend on the real traffic situations, the performance of drones, and the quality of controllers;

ii) this model does not take into account the advanced controllers.

The drone-following models do not describe a deterministic process, but they can be used as a stochastic process. To take into account the characteristics of advanced controllers, relative distance and actual reaction time of the controller are added to the control close-loop. This approach leads to an improved model called the Markov model.

The Markov model is based on the approximation of the stochastic process of velocity decision. One advantage over the SD model is that the inputs of the controller are different velocities and deviations in relative distance between the drones, which can be described as follows:

$$
\ddot{X}=f(\dot{X}, t)+\sigma(\dot{X}, t) \eta(t) .
$$

Using the Markov chain process, the developed model can be derived as follows: 


$$
\begin{aligned}
& \ddot{X}[k+1]=c_{v}\left(\dot{X}_{n-1}[k]-\dot{X}_{n}[k]\right)+ \\
& c_{x}\left[\left(X_{n-1}[k]-X_{n}[k]\right)-\Delta X_{\mathrm{pd} n}\right]+\varepsilon[k],
\end{aligned}
$$

where $\eta$ - the noise disturbing the process;

$c_{v}$ and $c_{x}$-coefficients which can depend on the time, given drone and controllers;

$\Delta X_{\mathrm{pd} n}=[\dot{X}(t)]-$ the predefined safe distance between the drones;

$k$ - the number of steps in a chain $(t=k \Delta t)$;

$\varepsilon(k)$ - the random value disturbing the process.

The improved model is called the Markov model. It should be noted that the first term on the right side of (4) represents the bias of velocities, while the second part describes the deviation of the predefined safe distance $\Delta X_{\mathrm{pd} n}$.

As the predefined safe distance is a nonlinear function, we can use a linear function in the developed model as follows: $\Delta X_{\mathrm{pd} n}=\beta V_{\mathrm{F}}$, where $V_{\mathrm{F}}=\dot{X}_{n-1}-$ the velocity of the following drone; $\beta$ - a positive constant.

Using discrete-time $z$ defined as $T=z \Delta t$, the acceleration on the left side of (4) can be substituted by $\ddot{X}(k+z)$. Therefore, the Markov model is referred to as being a discrete-time stochastic process, which can be improved by using:

i) parameters, $c_{v}$ and $c_{x}$, for relative velocities and distances;

ii) time reaction, $T$, depending on the actual relative distances, $\Delta X_{n}(k)$;

iii) controller's parameters depending on the real reaction time;

iv) estimated nominal values, $\Delta X_{\mathrm{pd} n}$ for the relative distances.

The improved model can be used for simulation and analysis of the chaotic processes that depend on the parameters applied in the drone-following models. In the present study, the delay time of the controller is the leading cause of the chaos in the drone-following process.

\section{RESULTS AND DISCUSSION}

The main results achieved in the numerical simulation experiments on the SD and the Markov models are provided in the present section. Besides, discussion and analysis based on these results are also provided.

In the SD model, it is assumed that the coefficient $\lambda$ is inversely proportional to the distance between the drones. Therefore, this coefficient is constant in the study.

The values of parameters used in the numerical simulation are shown in Table I.

TABLE I

THE PARAMETER VALUES

\begin{tabular}{|l|l|}
\hline \multicolumn{1}{|c|}{ Parameter } & \multicolumn{1}{c|}{ Value } \\
\hline Number of drones & 15 \\
\hline Initial velocity of drones & $20 \mathrm{~m} / \mathrm{s}$ \\
\hline Safe distance & $50 \mathrm{~m}$ \\
\hline
\end{tabular}

The values of the coefficients in the models are given in figures below. The effects of changes in these parameters and initial conditions are also analysed in the study.

Figure 2 demonstrates the acceleration of the leading drone.

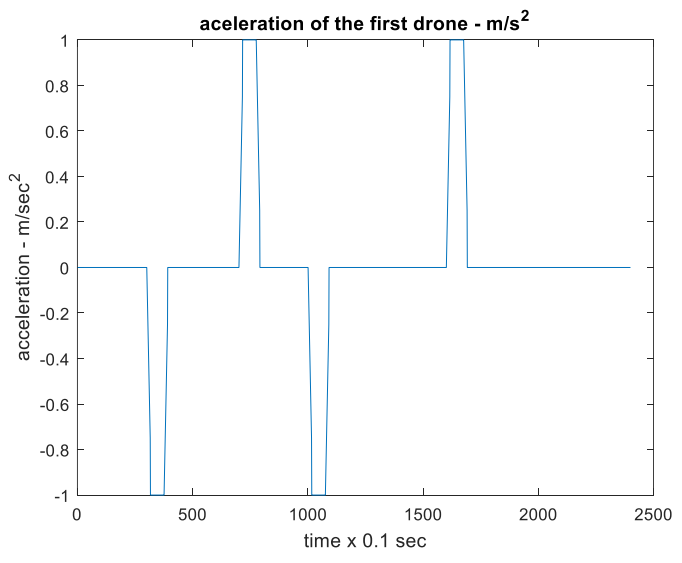

Fig. 2. The acceleration of the leading drone.

The following figures demonstrate the simulation results with SD and Markov models regarding the accelerations, velocities, relative velocities, distances, relative distances of followed drones. The model parameters are given in the figures.

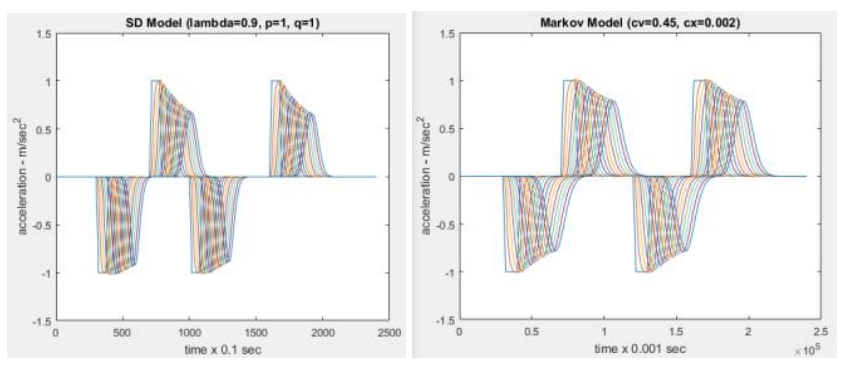

Fig. 3. The accelerations of the followed drones.
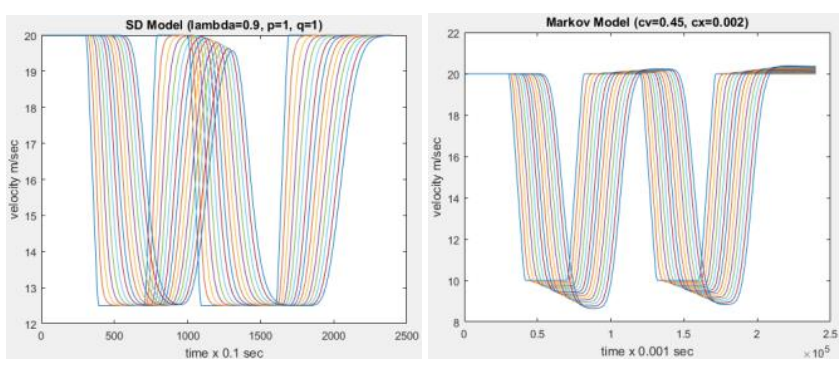

Fig. 4. The velocities of the followed drones.
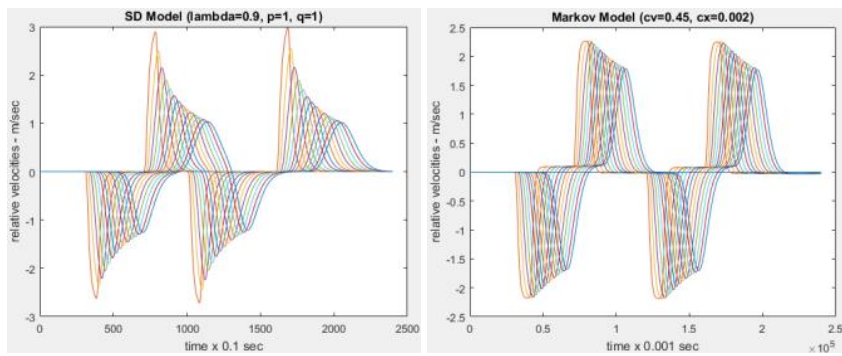

Fig. 5. The relative velocities of the followed drones. 

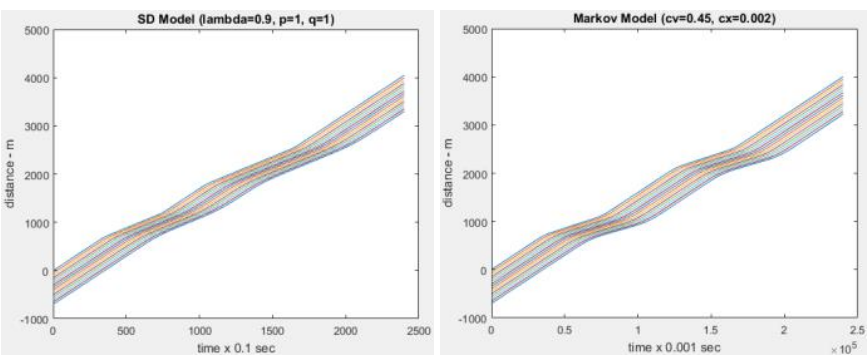

Fig. 6. The distances of the followed drones.
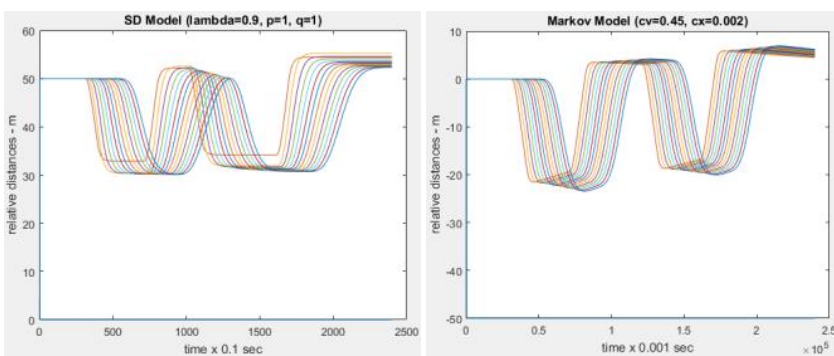

Fig. 7. The relative distances of the followed drones.

It can be noted that there is no accident and no unrealistic deceleration. The velocity of the followed drone is changed according to the speed of the drone ahead. However, the followed drone can react quickly compared to the reaction of the leading drone because of the difference in its acceleration. Such situations described the drone-following models precisely.

Even though the SD and the Markov models are quite similar, the reaction of the followed drone in the SD model is earlier than that in the Markov model. Besides, the dynamics of motion of the followed drone indicates that the stable state is slower in both models. In comparison with the SD model, the Markov model considers the changes in relative distance between drones. Moreover, in the Markov model, the more significant the variation of the velocity of the followed drone, the much smaller the relative distance between drones.

In the present study, the difference in parameters is introduced in order to investigate the performance of these models thoroughly. The results of numerical simulation, in this case, are shown in Figs. 8-12 for the SD model, and in Figs. 1317 for the Markov model.

Generally, the results show that when the parameter values are changed, the followed drones react slowly, which indicates that the time increment is increased. The impact of the SD model parameters on the results is shown in Table II.

TABLE II

IMPACT OF SD MODEL PARAMETERS

\begin{tabular}{|l|l|}
\hline \multicolumn{1}{|c|}{ Parameter } & \multicolumn{1}{c|}{ Result } \\
\hline Decreasing $p$ & $\begin{array}{l}\text { Accelerations and velocities of the followed } \\
\text { drones are decreased. These parameters are } \\
\text { changed slowly. } \\
\text { Time increment is increased. }\end{array}$ \\
\hline Changing $p, q$ together & $\begin{array}{l}\text { Accelerations and velocities of the followed } \\
\text { drones are changed quickly. } \\
\text { Time increment is reduced. }\end{array}$ \\
\hline Reducing $\lambda$ & $\begin{array}{l}\text { Velocities of the followed drones are decreased. } \\
\text { Time increment is increased. } \\
\text { Relative distances are increased. }\end{array}$ \\
\hline
\end{tabular}
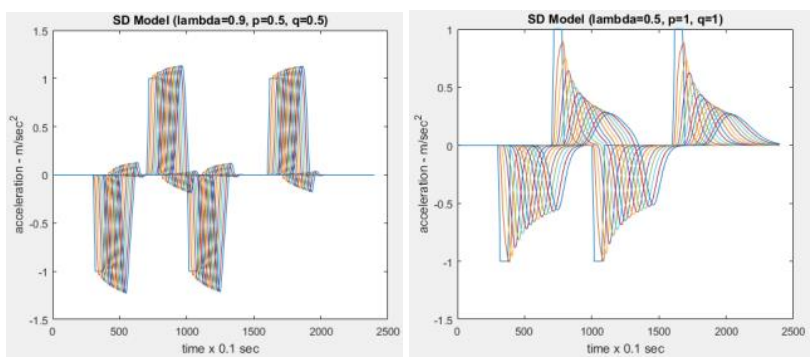

Fig. 8. The impact of SD model parameters on the acceleration.
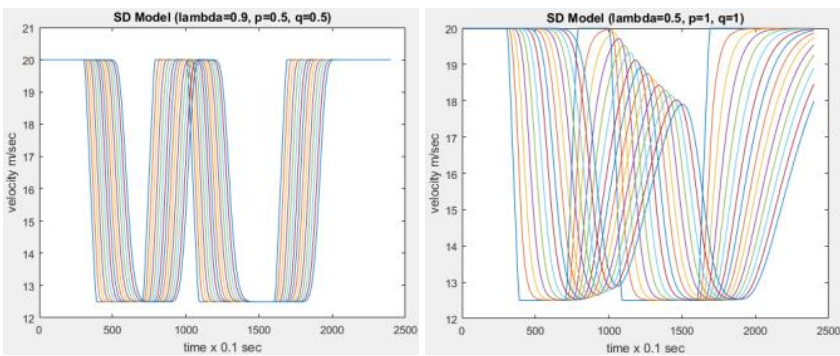

Fig. 9. The impact of SD model parameters on the velocity.
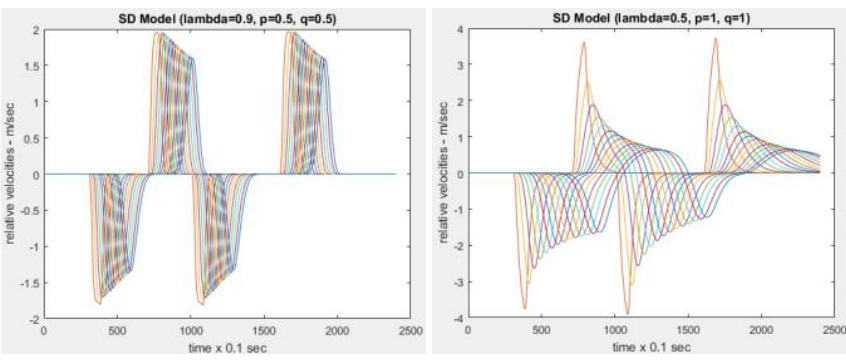

Fig. 10. The impact of SD model parameters on the relative velocity.
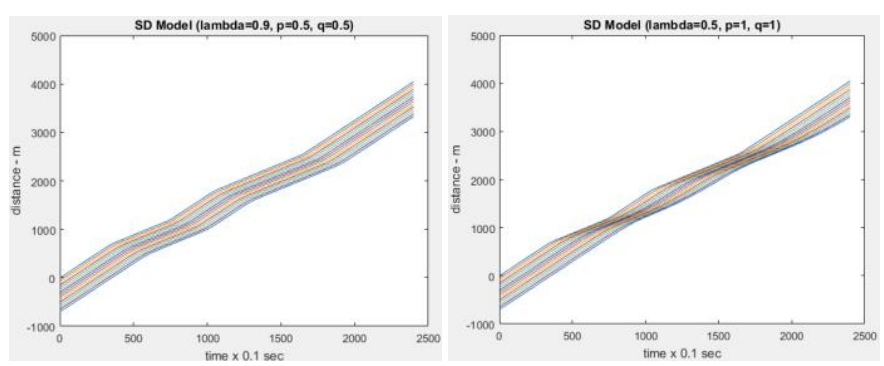

Fig. 11. The impact of SD model parameters on the distances.
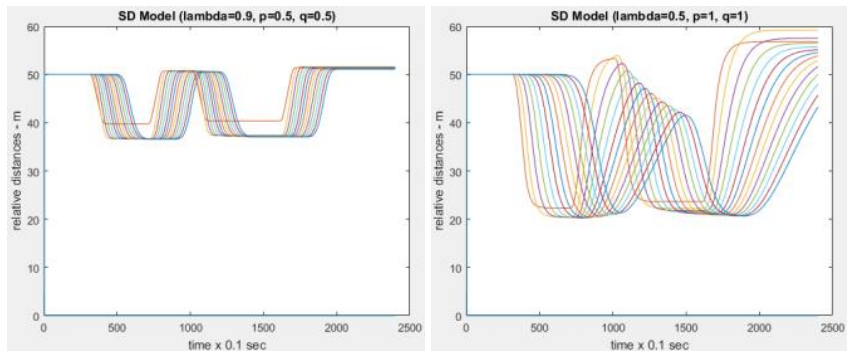

Fig. 12. The impact of SD model parameters on relative distances.

Compared to the Markov model, the results changed significantly. It can be seen in Figs. 13-17.

By observing the results, it can be concluded that the state of motion of the followed drone depends on the model coefficients. This dependence is shown by the changes of accelerations, velocities, distances, as well as of relative velocities and relative distances of the followed drones. 
2019, vol. 15 , no. 2

A summary of the impact of the Markov model parameters on the results is presented in Table III.

TABLE III

IMPACT OF MARKOV MODEL PARAMETERS

\begin{tabular}{|l|l|}
\hline \multicolumn{1}{|c|}{ Parameter } & \multicolumn{1}{c|}{ Result } \\
\hline Increasing $c_{v}$ & $\begin{array}{l}\text { Accelerations and velocities of the followed } \\
\text { drones are decreased. } \\
\text { Time increment is decreased. }\end{array}$ \\
\hline Increasing $c_{x}$ & $\begin{array}{l}\text { Relative velocities and relative distances are } \\
\text { reduced significantly. }\end{array}$ \\
\hline Changing $c_{\mathrm{v}}, c_{x}$ together & $\begin{array}{l}\text { Accelerations and velocities of the followed } \\
\text { drones are changed quickly. } \\
\text { Time increment is reduced. }\end{array}$ \\
\hline
\end{tabular}
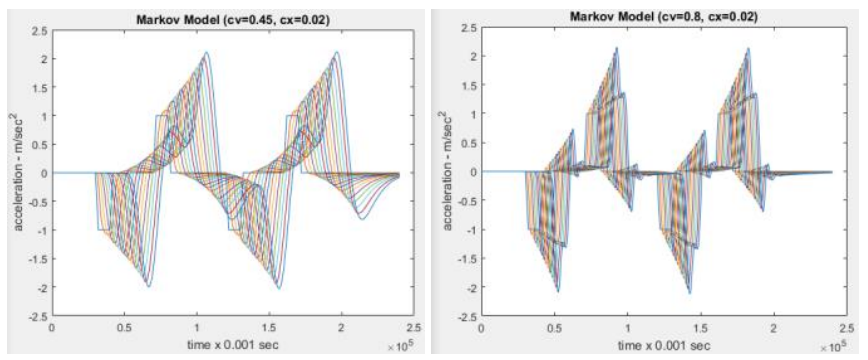

Fig. 13. The impact of Markov model parameters on the accelerations.
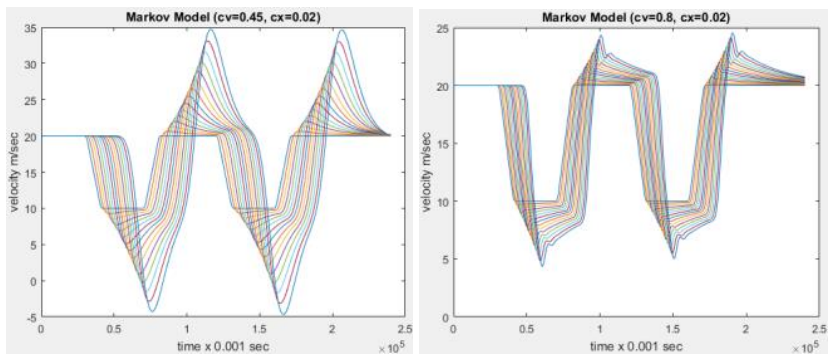

Fig. 14. The impact of Markov model parameters on the velocities.
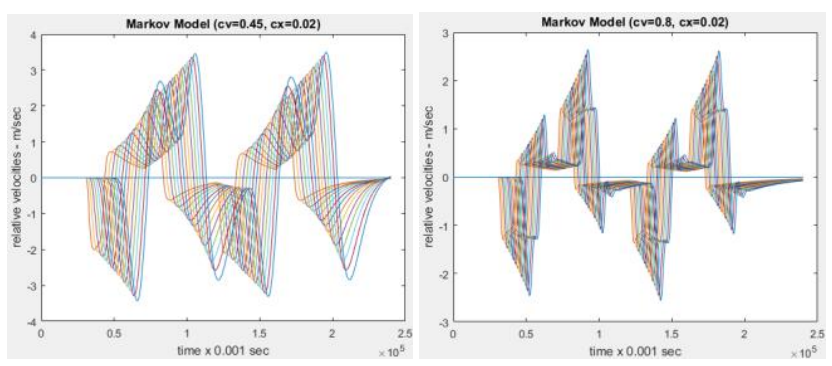

Fig. 15. The impact of Markov model parameters on the relative velocities.
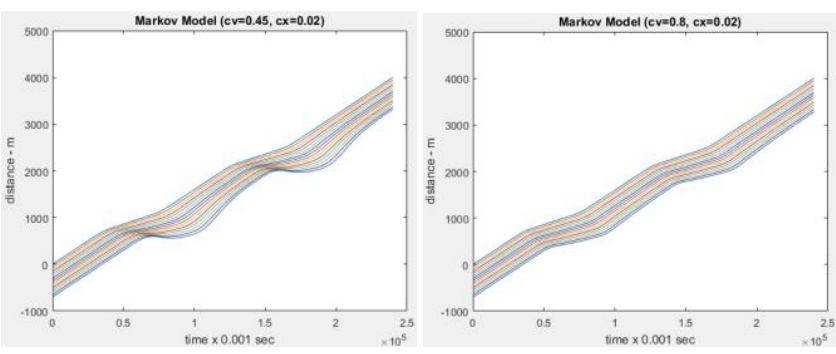

Fig. 16. The impact of Markov model parameters on the distances.

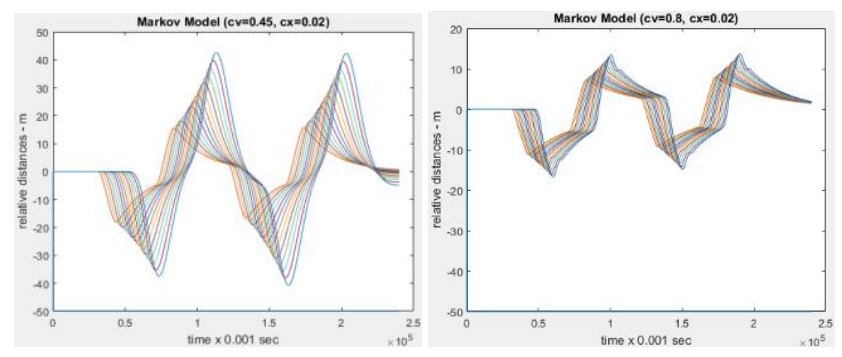

Fig. 17. The impact of Markov model parameters on the relative distances.

The results, in the study, also show the impact of the initial conditions, which can be seen in the figures above.
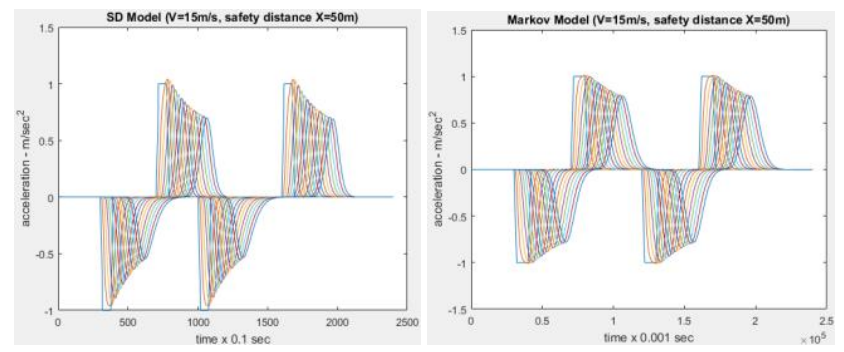

Fig. 18. The impact of the initial conditions on the acceleration.
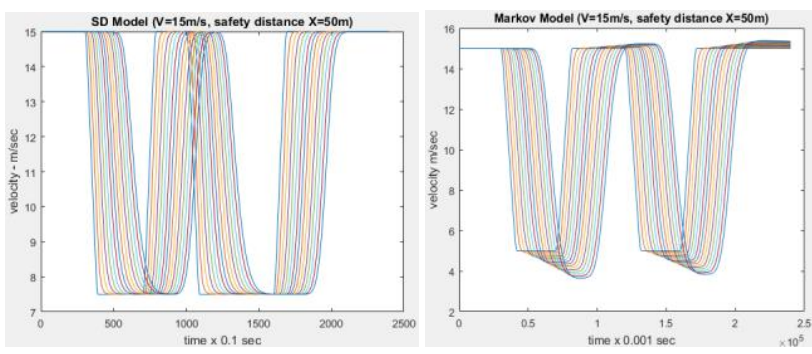

Fig. 19. The impact of the initial conditions on the velocity.
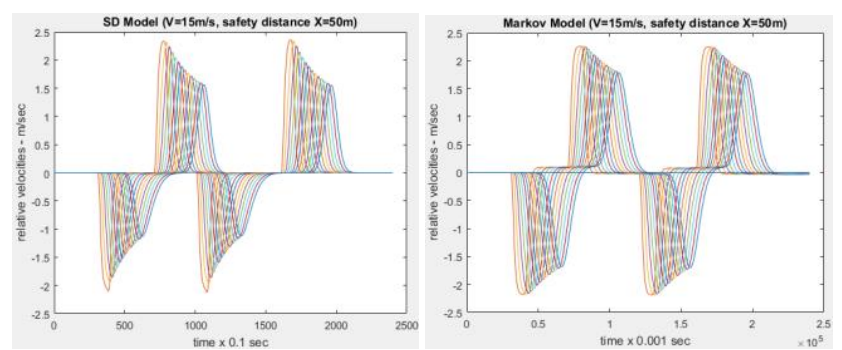

Fig. 20. The impact of the initial conditions on the relative velocity.
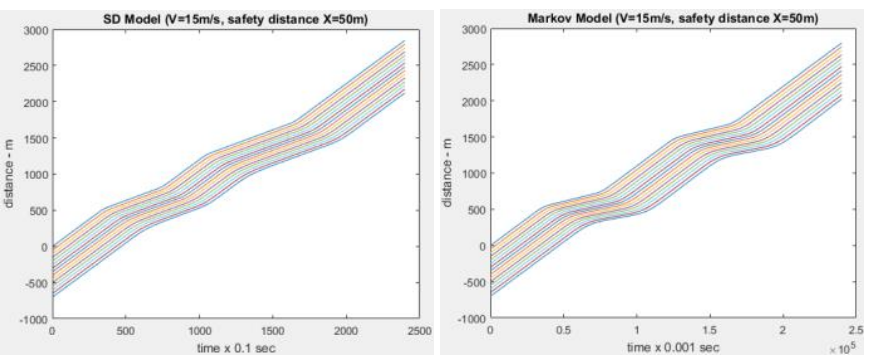

Fig. 21. The impact of the initial conditions on the distance. 


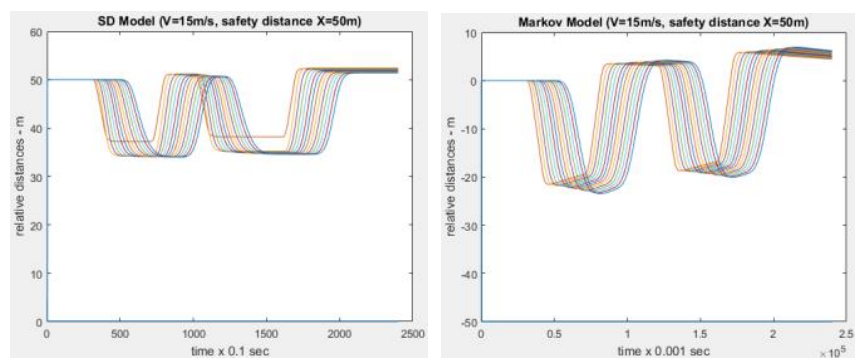

Fig. 22. The impact of the initial conditions on the relative distance.

It can be noted that the followed drones keep safe distance to drone ahead even if the velocities are changed. When the initial velocity is reduced, the changes in accelerations and velocities between drones in the SD model are significant than those in the Markov model. It indicates that the performance of the Markov model is better than that of the SD model.

Based on the simulation results and analysis above, several suggestions are given.

i) Safe distance is measured not only the drone directly in front but also two drones beside.

ii) The outputs of the controller are based on the estimation of the state of the system at a particular time, which can be used to control the followed drones.

iii) Several situations, such as the increasing number of drones, or one drone can get in or get out of the traffic flow, should be introduced to evaluate more accurately the performance of the SD model as well as the Markov model.

\section{CONCLUSION AND FUTURE RESEARCH}

We propose, in the present study, drone-following models for managing drones in the transportation management system in smart cities. These models are based on the initial idea that drones flight towards a leading drone in the traffic flow. The distance and relative velocity functions are used to describe such models.

Based on the numerical simulation results, it can be assumed that the safe distance between drones is maintained, which means that there is no accident in the traffic flow. While the SD model can be useful for studies of local stability, the performance of the Markov model is better than that of the SD model.

However, several situations should be continuously studied in the future. For instance, safe distance is measured not only in the drone directly in front but also in two drones beside. The second case is that the number of drones is increased. In addition, in order to obtain statistical estimates of certain functions and parameters for a preliminary evaluation of the mathematical models, it is necessary to design and conduct an experimental study to collect quantitative information regarding drone performance in space, in which one drone cannot pass another.

\section{REFERENCES}

[1] F. Castaldi, F. Pelosi, S. Pascucci, and R. Casa, "Assessing the Potential of Images from Unmanned Aerial Vehicles (UAV) to Support Herbicide Patch Spraying in Maize," Precision agriculture, vol. 18, no. 1, pp. 76-94, Aug. 2016. https://doi.org/10.1007/s11119-016-9468-3
[2] A. Capolupo, S. Pindozzi, C. Okello, N. Fiorentino, and L. Boccia, "Photogrammetry for Environmental Monitoring: The Use of Drones and Hydrological Models for Detection of Soil Contaminated by Copper," Science of the Total Environment, vol. 514, pp. 298-306, May 2015. https://doi.org/10.1016/j.scitotenv.2015.01.109

[3] P. Chamberlain, Drones and Journalism: How the Media is Making Use of Unmanned Aerial Vehicles. Routledge, 2017.

[4] A. Puri, "A Survey of Unmanned Aerial Vehicles (UAV) for Traffic Surveillance," Department of computer science and engineering, University of South Florida - internal report, pp. 1-29, Jan. 2005.

[5] C. A. Thiels, J. M. Aho, S. P. Zietlow, and D. H. Jenkins, "Use of Unmanned Aerial Vehicles for Medical Product Transport," Air Medical Journal, vol. 34, iss. 2, pp. 104-108, Mar. 2015. https://doi.org/10.1016/j.amj.2014.10.011

[6] F. Mohammed, A. Idries, N. Mohamed, J. Al-Jaroodi, and I. Jawhar, "UAVs for Smart Cities: Opportunities and Challenges," in Proc. 2014 Int. Conf. Unmanned Aircr. Syst. (ICUAS), 2014, pp. 267-273. https://doi.org/10.1109/ICUAS.2014.6842265

[7] E. Vattapparamban, İ. Güvenç, A. İ. Yurekli, K. Akkaya, and S. Uluağaç, "Drones for Smart Cities: Issues in Cybersecurity, Privacy, and Public Safety," in 2016 International Wireless Communications and Mobile Computing Conference (IWCMC), Sep. 2016, pp. 216-221. https://doi.org/10.1109/IWCMC.2016.7577060

[8] D. Wu, D. I. Arkhipov, M. Kim, C. L. Talcott, A. C. Regan, J. A. McCann, and N. Venkatasubramanian, 2016. "ADDSEN: Adaptive Data Processing and Dissemination for Drone Swarms in Urban Sensing," IEEE Trans. Comput., pp. 1-1, 2016 http://dx.doi.org/10.1109/TC.2016.2584061

[9] K. R. Sapkota, S. Roelofsen, A. Rozantsev, V. Lepetit, D. Gillet, P. Fua, and A. Martinoli, "Vision-Based Unmanned Aerial Vehicle Detection and Tracking for Sense and Avoid Systems," in 2016 IEEE/RSJ International Conference on Intelligent Robots and Systems (IROS), pp. 1556-1561, Oct. 2016. https://doi.org/10.1109/IROS.2016.7759252

[10] Y. Zhao and H. Pei, "An Improved Vision-Based Algorithm for Unmanned Aerial Vehicles Autonomous Landing," Phys. Procedia, vol. 33, pp. 935-941, 2012. http://dx.doi.org/10.1016/j.phpro.2012.05.157

[11] E. Vattapparamban, İ. Güvenç, A. İ. Yurekli, K. Akkaya, and S. Uluağaç, "Drones for Smart Cities: Issues in Cybersecurity, Privacy, and Public Safety," in 2016 International Wireless Communications and Mobile Computing Conference (IWCMC), Sep. 2016, pp. 216-221. https://doi.org/10.1109/IWCMC.2016.7577060

[12] "Commercial Drone Shipments to Surpass 2.6 Million Units Annually by 2025" [Online]. Tractica. Available:

https://www.tractica.com/newsroom/press-releases/commercial-droneshipments-to-surpass-2-6-million-units-annually-by-2025-according-totractica/ [Accessed: 21 Mar. 2019].

[13] R. M. Thompson, Drones in Domestic Surveillance Operations: Fourth Amendment Implications and Legislative Responses. Congressional Research Service, Library of Congress, Sep. 2012.

[14] International Civil Aviation Organization (ICAO), Unmanned aircraft systems (UAS), ICAO Cir 328 AN/190, 2011. Available: https://www.icao.int/Meetings/UAS/Documents/Circular\%20328_en.pdf [Accessed: 22 June 2019].

[15] International Civil Aviation Organization (ICAO), DOC 9859 AN/460 Safety Management Manual (SMM), 1st ed., 2006.

[16] Z. Sándor, "Challenges Caused by the Unmanned Aerial Vehicle in the Air Traffic Management", Periodica Polytechnica Transportation Engineering, vol. 47, iss. 2, pp. 96-105, Dec 2017. https://doi.org/10.3311/PPtr.11204

[17] T. Péter and K. Szabó, "A New Network Model for the Analysis of Air Traffic Networks", Periodica Polytechnica Transportation Engineering, vol. 40, iss. 1, pp. 39-44, 2012. https://doi.org/10.3311/pp.tr.2012-1.07

[18] G. Fedorko, V. Žofčinová, and V. Molnár, "Legal Aspects Concerning Use of Drones in the Conditions of the Slovak Republic Within the Sphere of Intra-Logistics", Periodica Polytechnica Transportation Engineering, vol. 46, iss. 4, pp. 17-84, Mar. 2018. https://doi.org/10.3311/PPtr.12131

[19] E. N. Barmpounakis, E. I. Vlahogianni, and J. C. Golias, "Unmanned Aerial Aircraft Systems for Transportation Engineering: Current Practice and Future Challenges," International Journal of Transportation Science and Technology, vol. 5, no. 3, pp. 111-122, Oct. 2016. https://doi.org/10.1016/j.ijtst.2017.02.001 
Nguyen Dinh Dung obtained the Bachelor and

Master degrees in control of aerial vehicles. He has been teaching at Le Quy Don Technical University since 2006. Currently, he is a $\mathrm{PhD}$ student at the Department of Aeronautics, Naval Architecture and Railway Vehicles, Budapest University of Technology and Economics, Hungary. He has been involved in several national and international studies and projects related to aerial vehicles, system design and control device integration of aircraft, concentrating on electronics, electrical engineering, and the assessment of environmental problems.

E-mail:ddnguyen@vrht.bme.hu

ORCID iD: https://orcid.org/0000-0002-8966-051X 